\title{
Assembly and annotation of genomes of some species from the apomictic genus Boechera and evolutionary analysis of apomixis-associated genes
}

\author{
V. Brukhin ${ }^{1,2 *}$, S. Kliver ${ }^{1}$, M. Rayko ${ }^{1}$, A. Komissarov¹, E. Bakin ${ }^{1}$, C. Rushworth ${ }^{3}$, \\ T. Mitchell-Olds ${ }^{4}$, U. Grossniklaus ${ }^{5}$ \\ ${ }^{1}$ Dobzhansky Center for Genome Bioinformatics, St. Petersburg State University, \\ St. Petersburg, Russia \\ ${ }^{2}$ Department of Plant Embryology \& Reproductive Biology, Komarov Botanical Institute \\ Russian Academy of Sciences, St. Petersburg, Russia \\ ${ }^{3}$ University and Jepson Herbaria, University of California, Berkeley, USA \\ ${ }^{4}$ Department of Biology, Duke University, Durham NC 27708-0338 NC, USA \\ ${ }^{5}$ Department of Plant \& Microbial Biology Zurich-Basel Plant Science Center, \\ University of Zurich, Zurich, Switzerland \\ *e-mail:vbrukhin@gmail.com
}

Key words: Boechera, Brassicaceae, genome, assembly, annotation, apomixis

Apomixis is asexual way of plant reproduction through seeds, which could be found in more than 400 plant species representing almost 40 families. It is believed that apomixis evolved independently in several taxa from sexual ancesters. Apomixis could be considered as a developmental variation of sexual reproduction in which some steps are lost, reduced, deregulated, desynchronized or changed. Thus, apomictic and sexual reproduction are closely related and they share many regulatory components. Molecular and genetic basis underlying apomixis and amphymixis (sexual reproduction) regulation still remains poorly understood. The ability to produce maternal clones and therefore to fix useful traits in the further generations of various crop plants could streamline agricultural breeding strategies based on the genetic aspects of apomixis. The potential of apomixis as a next generation technology for plant breeding attracts huge interest to elucidate molecular and genetic mechanisms of its regulation. Closely related to the model plant Arabidopsis thaliana, the genus Boechera is known to contain both sexual and apomictic species or accessions. Boechera retrofracta is a diploid sexually reproducing species and is thought to be an ancestral parent species of apomictic species. In the presentation will be reported the de novo assembly of the $B$. retrofracta genome using short Illumina and Roche reads from 1 paired-end and 3 mate pair libraries. The distribution of 23-mers from the paired end library has indicated a low level of heterozygosity and the presence of detectable duplications and triplications. The genome size was estimated to be equal $227 \mathrm{Mb}$. N50 of the assembled scaffolds was $2.3 \mathrm{Mb}$. 27048 protein-coding genes were predicted using a hybrid approach that combines homology-based and de novo methods. Also repeats, tRNA and rRNA genes were annotated. Finally, genes of $B$. retrofracta and 6 other Brassicaceae species were used for phylogenetic tree reconstruction. Also, we explored the histidine exonuclease $A P O L L O$ locus, related to apomixis in Boechera, and proposed model of its evolution through the series of duplications. An assembled genome of $B$. retrofracta will help in the challenging assembly of the highly heterozygous genomes of hybrid apomictic species such as $B$. divaricarpa. The $B$. retrofracta genome will also provide a basis to decipher the hybridogenesis events that led to the formation of apomictic Boechera accessions. 